\title{
Weak Discernibility and the Identity of Spacetime Points
}

\author{
Dennis Dieks \\ Institute for the History and Foundations of Science \\ Utrecht University, P.O.Box 80.010 \\ 3508 TA Utrecht, The Netherlands
}

\section{Introduction}

Science eschews concepts that are superfluous from an empirical point of view. As a case in point, science is averse to the use of the notion of haecceity, "primitive thisness", in order to individuate objects. Rather, objects as conceived in science derive their identity from their distinguishing qualitative properties, as defined by scientific theory. Science is thus sympathetic to Leibniz's principle of the identity of indiscernibles, with discernibility interpreted in terms of scientific concepts.

Natural as these ideas may seem, their application is not without difficulties. Even objects that are familiar from everyday life seem capable of sometimes violating Leibniz's principle - think of the case of Black's spheres, to be discussed below. The situation becomes only worse in fundamental physical theories like quantum mechanics and relativity. In the latter case, the problem is that spacetime points in symmetrical spacetimes (e.g., Minkowski spacetime) have all their properties in common, so that Leibniz's principle would lead to the absurd conclusion that there is only one point in such spacetimes.

In this article we shall further explain theses difficulties and then defend Leibniz'z principle, even for the symmetrical cases just mentioned. Part of our approach will be to invoke the notion of "weak discernibility", as proposed in recent literature. Weakly discernible objects share all their properties (both monadic and relational ones) but stand in irreflexive relations to each 
other - and on the basis of this it is possible to show that there are more than one of them. However, we shall argue that the application of this notion only achieves its aim if it is supplemented by a criterion of physical meaningfulness of the relations and relata in question; and that in the final analysis weak discernibility only helps out if it can be seen as a degenerate case of strong, absolute discernibility.

\section{The Identity Problem}

It is part and parcel both of ordinary life and the sciences to think in terms of different individual entities. Also both in ordinary life and the sciences, it seems obvious that empirical distinctions should accompany these differences in individuality -indeed, how could we have arrived at the concept of an object in the first place if not on the basis of such empirical differences?

In physics we accordingly expect that different individuals (different particles, for example) are characterized by different values of at least some physical quantities. With "physical quantities" we intend those general (qualitative) predicates that figure in physical laws and are thus relevant for empirical predictions - predicates like "mass", "charge", "velocity", etc. We could of course posit the individuality of objects by fiat, via the introduction of a notion of "primitive thisness" or "haecceity", so that each object comes to posses its own "thisness" that distinguishes it from all other objects. But these concepts do not do any work in physical theory and their introduction is therefore undesirable.

These expectations and intuitions are in agreement with the ideas behind Leibniz's principle of the identity of indiscernibles (PII). Roughly speaking, this principle says that two putative objects having all their properties in common are actually one and the same object. In order to be in accordance with what was just said, we have to restrict the domain of the properties considered in PII so as to include only physical quantities. If we take these quantities to include absolute position, as is reasonable within Newtonian mechanics with its absolute space, PII is fully satisfied by classical particles [7, Ch. 2]. Indeed, these particles are impenetrable so that they cannot occupy the same position in absolute space. Even if two Newtonian particles posses exactly the same mass,charge, and other physical attributes, they must still differ in where they are - and this is sufficient to ground their individuality in a physically acceptable way. 
However, on second thought complications arise, even for classical physics. The status of absolute space has been a bone of contention in the history of physics: it has been proposed repeatedly that space in itself is not a viable physical concept, and that we should rather think along the lines of Leibnizean relationism. According to the relationist position only the particles themselves exist, without being embedded in an independently existing space. The essential relationist idea is that the particles can nevertheless have spatial relations with respect to each other - these are possessed directly by the particles. Now, we do not need to take sides in the relationist-absolutist debate in order to acknowledge that relationism is a conceptual possibility. It seems commonsensical to accept the consistency of considering two (or more) particles that have distances etc. relative to each other without being pinned down in an absolute background space. But this conceptual possibility spells trouble for Leibniz's principle PII.

Indeed, even staying within the domain of everyday experience and without entering physical theory proper, it now seems possible to construct counterexamples to PII. These are cases in which it is obvious that there are several individuals, but in which there are no distinguishing qualitative characteristics, so that the individuals are indiscernible. One famous case, proposed by Max Black [2], is that of two perfect spheres of identical chemical composition and at a mutual distance of two miles in a relational universe (so there is no absolute background space). Another example is provided by a relational universe consisting of two hands that are each other's mirror images (Kant's enantiomorphic hands). The essential feature of these cases is that the objects have all their properties in common, both in the sense of monadic and relational predicates. Thus, both spheres in Black's example have the same material characteristics, and both are at two miles from a sphere. Similarly, Kant's hands each have the same internal geometric properties and both are mirror images of a hand. So these cases seemingly demonstrate that already in ordinary life we think in terms of concepts of object and individuality that are independent of the presence of distinguishing qualitative differences - in violation of PII.

The situation becomes even more urgent when we consider physical theory. A notorious obstacle for PII comes from quantum mechanics, in which the notion of "identical particles" plays a major role. These are particles of the same kind, i.e., with the same intrinsic properties (like mass, charge, spin); e.g., electrons, protons or neutrons. It is a basic principle of quantum mechanics that the state of a collection of such particles is completely 
symmetrical (in the case of bosons) or anti-symmetrical (fermions) with respect to the particle indices occurring in it. This symmetrization postulate implies that all one-particle states represented in the total state describing a collection of identical particles occur symmetrically in it: exchanging two one-particle states in the total state leaves the total state invariant (apart from a physically insignificant change of sign in the case of fermions). In other words, it does not matter which particle index we associate with which state. It follows from this symmetry that any property or relation that can be attributed, on the basis of the total quantum state, to any one particle has to be attributed to each of the other particles as well. Again, we are facing an apparent violation of PII.

The possible counterexamples to PII that will concern us most in this article are of a geometrical nature. To start with, consider the (infinite) Euclidean plane. This is a highly symmetrical geometrical object: it is invariant under translations, rotations and reflections. It follows that there are no privileged points in the Euclidean plane - indeed, symmetry transformations can transform any given point into any other, leaving all geometrical properties of these points and all mutual geometrical relations the same. This entails that each and every point in the plane possesses exactly the same geometrical status: the plane looks exactly the same viewed from whatever point. Clearly then, the points in the Euclidean plane fail to be discernible on the basis of their geometrical properties. If we assume that there are no haecceities that transcend geometrical properties, application of Leibniz's principle PII then appears to lead us to the (absurd) conclusion that there is only one point in the Euclidean plane.

The same argument can be given a more physical turn if we apply it to Newton's absolute space. However, let us immediately go on to special relativity theory and consider empty Minkowski spacetime. Special relativity posits four-dimensional Minkowski spacetime as the fixed geometrical background against which all physical processes evolve. Minkowski spacetime is endowed with a distance function: between any two points in it a definite distance is defined. The situation is more complicated, however, than in the Newtonian or Euclidean case: the special relativistic distance function can assume both positive and negative values, and moreover there are non-coinciding points between which the Minkowski distance is zero. From each point in Minkowski spacetime the total spacetime can accordingly be divided into three parts: all points that have a positive spacetime distance with respect to the chosen point (points with time-like separation); points 
at zero distance (points with light-like separation); and points that possess a negative distance (points with space-like separation) ${ }^{1}$. The points with timelike separation lie in the interior of the lightcone that can be drawn from the fiducial point in question; the points with space-like separation are outside of the lightcone; the lightcone itself consists of all points whose distance to the fiducial point vanishes. Because of this more involved metrical structure the symmetries of Minkowski spacetime assume a more complicated form than those of Euclidean space, but it remains nevertheless true that there are no privileged points. Minkowski spacetime looks exactly the same as seen from any spacetime point: it is completely homogeneous. It follows that all spacetime points have exactly the same geometrical properties - again, it seems impossible to ground their individuality in their geometrical features. PII then tells us that either there is only one point, or the individuality of the special relativistic spacetime points is of a haecceistic, non-geometrical nature.

The transition to general relativity does not alleviate this difficulty. First, the famous "hole argument" strengthens the idea that haecceities are incompatible with the physics of the situation. Indeed, if the existence of haecceities of spacetime points is accepted, this leads to an indeterminateness of the physical description: given one description, infinitely many other empirically equivalent descriptions can be given that differ only by the way they are positioned in the spacetime manifold. These descriptions differ by virtue of the haecceities of the spacetime points that are involved, but are exactly the same as far as their geometrical properties and empirical predictions are concerned. The hole argument can be summarized as the observation that if this numerical diversity of descriptions (and therefore also of solutions of the dynamical equations) is taken seriously, a radical indeterminism of physical theory has to be accepted: there are in this case infinitely many empirically equivalent evolutions, given any initial situation.

From a physical point of view these are all unnecessary complications: haecceities are superfluous from an empirical viewpoint and constitute a paradigm case of objectionable metaphysics. But their rejection has the consequence that we are compelled to look for another basis of the individuality of spacetime points, presumably by using PII.

But then our earlier problem repeats itself: also general relativity al-

\footnotetext{
${ }^{1}$ We employ the convention that time-like distances are positive; in the literature the opposite convention that makes space-like distances positive is also sometimes used.
} 
lows spacetimes with a high degree of symmetry. Minkowski spacetime, the special case of special relativity contained in general relativity, is only one example. The standard cosmological models, the so-called FriedmannLemaitre-Robertson-Walker (FLRW) spacetimes, furnish further instances. These spacetimes allow the introduction of a cosmic time scale, at each instant of which three-dimensional space is completely homogeneous. Repetition of the earlier argument based on PII then leads to the conclusion that at each instant of cosmic time there is only one spatial point [18]. Evidently, this conclusion conflicts with the way general relativity itself describes these cosmological models.

\section{Weak Discernibility}

As Hawley points out [9], defenders of Leibniz's principle can respond to such putative counterexamples to PII in a variety of ways. First, they may query whether the described situations are possible at all-this does not appear to be a promising way out in our above examples, which are all possible according to the relevant physical theories. Second, defenders of PII can dispute that these situations are best described in terms of distinct but indiscernible individuals. This can take two forms: either it may be argued that a correct analysis of discernibility will reveal that the objects in question are discernible after all, or it may be claimed that it was a mistake to assume that there were distinct objects to start with - that there is actually only one undivided whole.

The latter option should certainly be taken seriously. It can be argued that the quantum case (the case of "identical quantum particles") calls for exactly this response: there are no individual fermions and bosons but there is rather only one undivided quantum field $[3,4,5]$. Later we shall say a bit more about the justification for thinking this. However, in the geometrical cases, and in the cases of Black's spheres and Kant's hands, there are good reasons for going another way. As we shall argue, here we are entitled to think that we are in fact dealing with separate objects and that a more refined analysis of discernibility can show this to be justifiable by PII.

In order to explain this more refined analysis we follow Saunders [14, 15], who takes his cue from Quine [13], in noting that in cases like the ones mentioned above irreflexive relations are instantiated: i.e., relations that entities cannot bear to themselves. Thus, Black's spheres are at a non-zero distance 
from each other - but a sphere cannot be at a non-zero distance from itself ${ }^{2}$; Kant's hands are each other's mirror images - but a hand cannot be its own mirror image; and the points in the Euclidean plane have distances with respect to each other that they cannot possess with respect to themselves. Similarly, in each of the spaces-at-an-instant in the FLRW cosmological models general relativity defines a spatial distance function such that points do not have non-zero distances with respect to themselves (at least as long as one restricts oneself to spatial regions that are not too large - since these spaces may be closed, the situation in the large may be analogous to that of points on a circle). More generally, in arbitrary relativistic spacetimes irreflexive relations between points can be constructed on the basis of the four-dimensional distance function that relativity theory defines on such spaces (see also [11]). In the case of points with space-like or time-like separation this is obvious: in this case there is a positive or negative distance between the points that cannot exist between any point and itself (at least not within regions that are not too extended). The prima facie more complicated case of points with light-like separation (i.e. with four-distance zero) can be accommodated by observing that any two distinct points that are light-like separated can be connected via the combination of a non-zero time-like and a non-zero spacelike interval ${ }^{3}$, something which cannot hold for any point with respect to itself.

The irreflexivity of these relations is the key to proving that (a generalized version of) PII is satisfied after all: if an entity stands in a relation that it cannot have to itself, there must be at least two entities.

To see in detail how this works, let us formalize the argument. PII can be formulated as follows, with = denoting identity:

$$
s=t \equiv \forall P(P(s) \leftrightarrow P(t)) .
$$

The universal quantifier here ranges over all physical predicates $P$ (not haecceities!). The right-hand side of the equation stipulates that $s$ and $t$ can replace each other, salva veritate, in any $P$.

There can now be various kinds of discernibility ([15]). Two objects are absolutely discernible if there is a one-place predicate that applies to only one

\footnotetext{
${ }^{2}$ We are here taking it for granted that the distance relations satisfy Euclidean geometry. If, instead, the distances satisfied the geometry of a three-sphere, or another closed space, the argument given below for the numerical diversity of the spheres could fail.

${ }^{3}$ Consider two points $P$ and $Q$, such that the four-distance $\sigma(P, Q)=0$. Then there exists at least one point $R$ such that $\sigma(P, R)>0$ and $\sigma(R, Q)<0$.
} 
of them; relatively discernible if there is a two-place predicate that applies to them in only one order; and weakly discernible if an irreflexive two-place predicate relates them. The latter possibility is relevant to our examples. If there is an irreflexive but symmetric two-place predicate $P(.,$.$) that is$ satisfied by $s$ and $t$, the definition (1) requires that if $s$ and $t$ are to be identical, we must have:

$$
\forall x(P(s, x) \leftrightarrow P(t, x))
$$

But this is false: in any valuation in which $P(s, t)$ is true, $P(t, t)$ cannot be satisfied since $P$ is irreflexive. It follows that PII is satisfied by any two non-identical objects that stand in an irreflexive physical relation.

In our cases with irreflexive relations PII is therefore sufficient to ground the numerical diversity of the objects after all. It should be noted, however, that although weak discernibility is thus able to lay a non-haecceistic fundament under numerical diversity, this does not endow the objects with individuality in the usual sense. Indeed, in the situations we have discussed it remains impossible to pick out or define any single object. Because of the symmetry any property or relation that can be attributed to one object can equally be attributed to any other and we can therefore not identify any specific object. It is impossible, for example, to pin down any particular point in the Euclidean plane on the basis of the properties of the plane and its points, even if we include all relational properties (of course, we are speaking here of the plane tout court, without adding by hand a preferred point that could function as an origin).

This lack of identifiability may raise doubts on the meaningfulness of the claim that there are more than one separate objects after all (cf. [10]). However, in order that the number of elements in a domain is a well-defined quantity it is sufficient that a function exists that maps the domain oneto-one onto a set of labels, e.g. the set $\{1,2, \ldots, n\}$; it is not required that we can actually construct such a labelling. In the examples we have been considering it was actually given in the description of the cases (two spheres, two hands, many points) that such mappings exist, and the question to be answered was simply whether this mapping could be given a basis via PII. And as we have seen, this can be done with the help of irreflexive relations. 


\section{Scientifically Respectable Objects and Re- lations}

It is important, of course, that the irreflexive relations we consider here are scientifically respectable. If we started by just stipulating that a certain domain is labelled by a set of natural numbers, between which there are irreflexive relations, this would be completely empty. Labels can always be posited abstractly, and there are always irreflexive relations between them; for example, the irreflexive relation of "being unequal to each other" between the natural numbers. Proceeding this way we would do nothing to make it acceptable that the domain in question really splits up and consists of physical objects. The numbers would in this case function as haecceities in disguise, without scientific merit.

The possibility of "splitting up a domain" is certainly not a priori evident in the context of discussions about whether or not PII applies. As we have seen at the beginning of the previous section, one possible stance in such discussions is to argue that there is no multiplicity at all: that there is only one undivided physical system. No questions about the individuation of elements of the domain by means of PII have to be answered if there is only one system, and a parsimonious ontological picture results. If there is no convincing reason to think of the domain as consisting of several separate entities in the first place, this kind of holism recommends itself [8]. As stressed before, what we need in order to think of numerical diversity is an argument that the objects and the relations between them are scientifically respectable and latch on to the structure of the domain as described by physics.

It may be the case that there is nothing else to characterize the objects in the domain than the relations they stand in. This possibility has been worked out in structuralism, where objects are viewed as nodes in a relational network (this is compatible with the idea that the relations in their turn can only exist if they connect actual relata [6]). So if we are going to verify scientific respectability we need not necessarily assume that there is a division of labor between "objecthood providers" that must be checked first and relations that only become relevant later. A relational structure may constitute our only access to the existence of entities - in fact, this is the situation we shall encounter in the case of spacetime points. 


\section{Spheres, Hands, Euros and Other Objects}

Looking back at the examples of Black's spheres and Kant's hands, we see that the objects there (spheres and hands, respectively) were characterized independently of the relations that hold between them. The spheres were stipulated to be of of a certain chemical composition (pure iron, says Black), and of course they possess a definite geometrical shape. We know very well what such spheres are like: they are doubtlessly physical objects. Similarly, we are familiar with hands and do not doubt their status as physical entities. The difficulty of these cases is not in deciding whether there are objects at all, but rather in finding a basis for their numerical diversity: since the physical characteristics are completely equal for both objects, PII seems to suggest that there is actually only one of them. The information that an irreflexive relation holds (being at a two-miles distance from each other and being each other's mirror images, respectively) now helps out: application of PII demonstrates that there must be two objects - although the perfect symmetry of the situation makes them only weakly discernible.

The uncontroversial physical nature of the irreflexive relations in question is relevant here. For when we allow relations that refer to "fantasy predicates", not sanctioned by physics, there is no limit to the number of entities that may result. One might for instance imagine that in every perfect iron sphere a "mork" and a "gork" copy of this sphere coincide, and that since these two qualities exclude each other (an irreflexive relation) we have actually two spheres. This example is simplistic and far-fetched, but the point still stands that we should make sure that the relations we are considering are bona fide from a scientific point of view if we wish to draw conclusions about the number of scientifically respectable objects. If the relations are our only access to the objects, it becomes even more pressing to verify their scientific status.

A well-known more realistic example of irreflexive relations without there even being any actual objects at all is the case of money in a bank account (not coins in a piggy bank, but transferable money in a real bank account). Imagine an account with five Euros. It is easy enough to speak about this money as a collection of five entities, and a mapping of the set $\{1,2,3,4,5\}$ to the account can be defined in an abstract way (e.g., draw a circle representing the total amount and divide it into 5 equal parts; or think of the mapping as given by the order in which amounts of one Euro were transferred to the account), but this does not prove anything about the actual presence of five 
individual entities in the account. On the contrary, the case of more than one money units in a bank account is the standard example of absence of individuality; it is a case in which only the account itself, with the total amount of money in it, can be treated as possessing individuality [16, 17]. Although we are accustomed to using relations and things talk in this case (e.g., "the last Euro that has come in is more important than the other ones"), this talk does not represent the actual physical situation (it may represent aspects of how that situation came about; but that is not our concern here).

A (more controversial!) example from present-day science comes from quantum mechanics. A notorious interpretational issue in this theory is the status of so-called "identical particles": are these "particles" individual entities or should they rather be described in a holistic way (in terms of one field)? The theory suggests that there are distinct entities because it works with indices $1,2,3, \ldots, n$ that label the one-particle Hilbert spaces (the quantum mechanical state spaces) that can be used to construct the total state space of the system. However, it is a general principle of quantum mechanics that the state defined in this total space must be completely symmetrical (the case of "bosons") or anti-symmetrical ("fermions") in these indices. This symmetrization postulate implies that if the total state is restricted to any of the one particle state spaces the result is exactly the same for all indices. It follows that if these indices are to represent particles, any property or relation that may be attributed to any one of these putative particle is attributable to each of the others as well.

One response is to take this as a signal that there are no different particles at all: although there is talk about the indices in the formalism in terms of particles, this should be understood in the same way as talk about the different Euros in a bank account. From a fundamental point of view it is better, according to this line of reasoning, to renounce talk that suggests the existence of individual particles and to reconceptualize the situation in terms of the excited states of a field (analogous to thinking of the Euros in an account as one sum of money). This response leads into the direction of quantum field theory.

However, the situation is also reminiscent of Black's spheres and Kant's hands. As we have seen there, symmetry is not decisive for proving the absence of Leibniz-style individuality: we may be facing a case of weak discernibility. Perhaps there are irreflexive physical relations between the fermions and bosons that guarantee their (weak) individuality in the same way as they did for Black's spheres and Kant's hands $[12,15]$. Now, we have already seen 
that in the total state indices $1,2,3, \ldots, n$ occur; and it is certainly possible to define irreflexive relations between them (see $[3,4,5,12,15]$ for details). But does it follow that these indices correspond to weakly discernible entities?

\section{To Be or Not to Be}

Obviously, we know that spheres and hands are bona fide physical objects: we possess direct experience with objects of this kind. But let us reflect for a moment about the background of our certainty in these cases. We are familiar with collections of more than one spheres and hands in asymmetrical situations, where it is possible to uniquely distinguish and name them. Typically, we think of two hands or two spheres as placed differently with respect to ourselves as observers: one being at our left and one at our right, for example. The possibility of these asymmetrical configurations gives us confidence about the nature of spheres and hands even if they are placed in a completely symmetrical situation. They are clearly entities of which several copies can exist next to each other. The symmetrical configurations in which they are only weakly discernible are limiting cases of more typical asymmetrical situations in which a point of reference, an observer, or something similar is given, and in which they are absolutely discernible.

Likewise, the typical context in which classical particles occur is that of asymmetrical situations, in which the network of mutual distances suffices to characterize each individual particle in an unambiguous way. Changing the mutual distances so that the configuration becomes more symmetrical will evidently do nothing to the nature of the objects: as long as the situation is only slightly asymmetrical they will remain absolutely discernible physical entities, whereas in the limiting case of complete symmetry they still are the kind of entities that are candidates for absolute discernibility. There is no indication in physical theory or anywhere else that there might be ontological changes in the objects just because of approaching a fully symmetrical Black's spheres-type configuration.

The (standard, absolute) discernibility in asymmetrical situations, plus the possibility of a limiting procedure, thus provides us with a test for physical relevance and physical objecthood in symmetrical situations. We are justified in assuming the existence of actual entities (candidate individuals) if the breaking of the symmetry is physically possible, does not involve any change in the type of properties assigned, and results in a situation with 
distinguishable objects (this strategy resembles the one followed by Adams, who proposes to compare Black's spheres with spheres of which one has a very slight chemical impurity [1, p. 17]).

These cases are to be contrasted with the case of the Euros in a bank account. If we start with actual Euro coins, there is no limiting procedure by means of which we can gradually approach Euros as units of transferable money. There is consequently no argument here that bank account Euros and Euro coins are ontologically similar. On the contrary, according to our best available way of describing what is going on in bank accounts, there is only a total amount of money in them, not composed of individual Euros.

To investigate into which category "identical quantum particles" fall, the transferable Euro or the Black's spheres one, we can try and copy the strategy followed in the classical particle case, namely breaking the symmetry and seeing whether absolutely discernible entities result. But here we run into a difficulty of principle: quantum mechanics forbids "identical particle" systems that are not in a fully (anti-)symmetric state - it is a matter of quantum mechanical law that "fermions" and "bosons" can only have exactly the same states and relations. (A more accurate statement would be that the indices occurring in the formalism must always be completely interchangeable as far as physical features of the state are concerned.) This is significantly different from the symmetrical classical cases, in which the symmetry was contingent and the theory allowed evolutions from symmetrical to asymmetrical configurations. In quantum mechanics the mutual relations between fermions cannot serve to distinguish individual component systems as a matter of principle, and our earlier test fails.

It might be replied that this by itself does not yet prove that there are no individual identical quantum particles - indeed, we could also imagine a hypothetical classical world in which a law stipulates that perfect spheres can only occur in completely symmetric configurations. But in such a world we could still have good reasons to think in terms of individual spheres: our theories could allow for an external object serving as a point of reference that makes the spheres discernible (e.g., an observer who stands in different relations to the various spheres). If no argument of this kind were to be possible at all, the existence of individual spheres would surely become moot. However, this is precisely the situation that obtains in quantum mechanics. It follows from the quantum formalism, as a matter of law-like principle, that "identical quantum particles" have exactly the same relations with respect to any external vantage point that may be introduced $[3,4]$. 
More can and should certainly be said about the quantum case (cf. [5]) the subject remains controversial. But the above should suffice to show that it is not at all evident that "identical quantum particles" should be conceived of as numerically diverse objects.

\section{The Status of Spacetime Points}

Where does this lead us with respect to the question of whether spacetime points are individuals - and if so, in what way their individuality can be grounded? In section 2 we have encountered the dilemma that the points in the Euclidean plane have all their monadic and relational properties in common, as a consequence of the perfect homogeneity of the plane. If we wish to avoid haecceities and try to ground the individuality of geometrical points in their geometrical properties, this appears to lead to the conclusion that there cannot be more than one point in the plane. The situation does not seem to improve when we go from mathematics to physics: in Minkowski spacetime, but also in symmetrical solutions of the field equations of general relativity (e.g., the FLRW models), spacetime points share again their properties and the conclusion that on a non-haecceistic account this entails that the universe consists of one lonely point has indeed been drawn in the literature ([18] - the author of this article intends this as a reductio of a non-haecceistic structuralist position).

As we have explained in section 3, the notion of weak discernibility can here come to the rescue. Both in the case of mathematical geometries and in the case of physical (spacetime) geometries irreflexive relations exist between the points. If these relations are admitted to the domain of relations used in the application of Leibniz's principle PII, logic dictates that a numerical diversity of weakly indiscernible objects exists, exactly as needed.

However, we should not be too quick. As we have seen illustrated in sections 4,5 and 6 , we need some guarantee that the thus defined weakly discernible objects, and the relations between them, are scientifically respectable: we should restrict the domains of quantification in PII so as to avoid artificial predicates and properties.

When we look again at the mathematical example of Euclidean geometry (in a modern axiomatization), the first thing to observe is that the geometrical points here are not assumed to possess any properties except for what is fixed by the relational structure to which they belong. It is possible to 
axiomatize the full geometry of the plane by describing it as a manifold of elements on which a non-negative distance function is defined. So what we have here is the structuralist possibility already alluded to at the end of section 4, in which our only access to objecthood is provided by the relations between the objects. In the present case these relations are mutual distances - and since distances greater than zero constitute irreflexive relations we recover the usual structure of the plane with its infinity of points as weakly discernible objects. As long as we are discussing the plane as a purely mathematical structure, the question of whether the distance relations are significant or not and of whether it would not be better to describe the plane as one undivided whole, does not arise: within mathematics the relational structure is just posited to exist (either as being defined by us or as abstractly existing in a mathematical sense) and studied as such.

The interesting questions pertain to physical respectability - even if a numerically diverse mathematical structure is perfectly alright in itself, it can still be asked whether its application to physical reality is justified. So consider a physical counterpart to the Euclidean plane, namely the completely homogeneous three-dimensional space that occurs in Newtonian physics. Here also all points have exactly the same status, and can be understood as specified by the structure of distance relations in which they are the relata. This leads again to an (uncountable) infinity of weakly discernible points. By contrast to the purely mathematical case, however, we may now raise the question whether the network of distances, and the points in it, are really physically significant.

At first this may seem an absurd question: what could be more physically significant than distances? However, we were considering empty Newtonian space, without material contents. That means that there are no measuring rods or physical processes that "feel" the distances. The introduction of such material things would generally destroy the homogeneity that we have assumed, and would lead to a completely different situation. Now, if Newtonian physics were nothing but the study of empty Newtonian space, the numerical diversity of the points in this space would fulfil no physical role. In this case we would have no guarantee that the relational structure and the points in it correspond to anything physical, and it would be fully justified to think of space as one undivided entity. In other words, if only a completely empty homogeneous space were physically possible, its internal structure would have no obvious physical meaning. It could be stipulated to exist, of course, but its status would be mathematical or metaphysical rather 
than physical.

But this is evidently not at all the predicament we are actually in. Reallife applications of Newtonian physics deal with situations that are not even nearly symmetrical, because they assume many objects with unequal properties in asymmetrical configurations. In such situations it generally becomes possible to discern the spatial points absolutely, with the help of distances and angles with respect to the objects that are present. This is analogous to what happens when we add an origin and coordinate axes to the Euclidean plane. The completely symmetrical homogeneous case thus appears as an idealization, a limiting case of further and further abstracting from actual asymmetries. If we look at all physically possible cases in Newtonian space there can be no doubt about the physical significance of the absolutely discernible points, because the distinguishing relations will in some cases be actually physically instantiated (by means of measuring rods, for example).

The physical significance of the relations and relata in the fully symmetrical cases thus follows from a comparison with other possible cases-put differently, there is a modal aspect involved (where the modality is physical, determined by physical theory).

Completely similar observations can be made for special and general relativity. If special relativity were exclusively a theory about empty Minkowski spacetime, the physical significance of spacetime points in the theory would be doubtful at most. And if general relativity were solely about completely symmetrical FLRW universes the same would be true here. But in fact empty Minkowski spacetime and the completely homogeneous FLRW cosmological models are degenerate cases, that can be connected to more realistic, asymmetric spacetimes via a limiting process. This is just like what we saw in the examples of Black's spheres and Kant's hands: the physical significance comes from a comparison with asymmetric situations.

\section{Conclusion}

In completely symmetrical situations Leibniz's principle PII seems prima facie unable to ground the individuality and numerical diversity of objects. The notion of weak discernibility, using irreflexive relations, appears to come to the rescue: although it cannot yield individuality in the ordinary sense of identifiability, it is able to ground the numerical diversity of objects. In particular, this seems to offer a way out to the problem of how to individuate 
points in space or spacetime.

What we have argued in this article is that we need a criterion to ensure that the weakly discernible objects that thus result are scientifically respectable. In order to verify this we have to make sure that the irreflexive relations upon which the weak discernibility hinges are scientifically relevant. In particular, in the space and spacetime cases we need an argument that the mutual distances between the points are physically significant. As it turns out, this physical significance depends on the possibility, according to physical theory, of situations in which the symmetry is broken: we can only have empirical access to single objects (here: points) via relations that make a distinction between different objects. If this is correct, the relevance of the irreflexive relations used to establish weak discernibility depends on their ability to ground absolute discernibility in asymmetrical situations. Consequently, weak discernibility only helps out if it can be regarded as a degenerate case of absolute discernibility.

\section{References}

[1] Adams, R.M.: Primitive Thisness and Primitive Identity. Journal of Philosophy 76, 5-26 (1979)

[2] Black, M.: The Identity of Indiscernibles. Mind 61, 153-164 (1952)

[3] Dieks, D. and Versteegh, M.A.: Identical Quantum Particles and Weak Discernibility. Foundations of Physics 38, 923-934 (2008)

[4] Dieks, D.: Are 'Identical Quantum Particles' Weakly Discernible Objects? Chapter 3 in Suárez, M., Dorato, M. and Rédei, M. (eds.) EPSA, Philosophical Issues in the Sciences, Vol. 2. Springer (2010)

[5] Dieks, D. and Lubberdink, A.: How Classical Particles Emerge From the Quantum World. Foundations of Physics, to appear (2011). DOI: 10.1007/s10701-010-9515-2

[6] Esfeld, M. and Lam, V.: Moderate Structural Realism about SpaceTime. Synthese 160, 27-46 (2008); Ontic Structural Realism as a Metaphysics of Objects. Chapter 8 in Bokulich, A. and Bokulich, P. (eds.), Scientific Structuralism. Springer (2010) 
[7] French, S. and Krause, D.: Identity in Physics: A Historical, Philosophical, and Formal Analysis. Oxford University Press (2006)

[8] Hawley, K.: Weak Discernibility. Analysis 66, 300-303 (2006)

[9] Hawley, K.: Identity, Indiscernibility and Number. Unpublished manuscript (2007)

[10] Keränen, J.: The Identity Problem for Realist Structuralism. Philosophia Mathematica 9, 308-330 (2001)

[11] Muller, F.A.: Structuralism and the General Theory of Relativity. Unpublished manuscript (2010)

[12] Muller, F.A., and Seevinck, M.: Discerning Elementary Particles. Philosophy of Science 76, 179-200 (2009)

[13] Quine, W.V.: Grades of Discriminability. Journal of Philosophy 73, 113116 (1976). Reprinted in Quine, W.V.: Theories and Things. Harvard University Press (1981)

[14] Saunders, S.: Physics and Leibniz's Principles. In Brading, K. and Castellani, E. (eds.) Symmetries in Physics: Philosophical Reflections. Cambridge University Press (2003)

[15] Saunders, S.: Are Quantum Particles Objects? Analysis 66, 52-63 (2006)

[16] Schrödinger, E.: Science and Humanism. Cambridge University Press (1952). Partly reprinted as: What is an Elementary Particle? In Castellani, E. (ed.) Interpreting Bodies: Classical and Quantum Objects in Modern Physics, pp. 197-210. Princeton University Press (1998)

[17] Teller, P.: Quantum Mechanics and Haecceities. In Castellani, E. (ed.) Interpreting Bodies: Classical and Quantum Objects in Modern Physics, pp. 114-141. Princeton University Press (1998)

[18] Wütrich, C.: Challenging the Spacetime Structuralist. Philosophy of Science 76, 1039-1051 (2009) 